\title{
Number of Years When Consumed More than 2 Drinks per Day for Men and More than 1 Drink per Day for Women
}

National Cancer Institute

\section{Source}

National Cancer Institute. Number of Years When Consumed More than 2 Drinks per

Day for Men and More than 1 Drink per Day for Women. NCI Thesaurus. Code C156824.

The number of years an individual has consumed more than 2 drinks per day for men and more than 1 drink per day for women. 